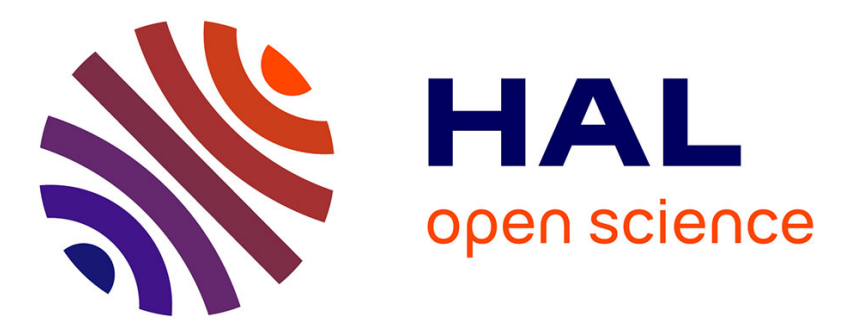

\title{
Comment concilier l'inconciliable: la place concevable de l'ethnologie dans le musée de la Cité nationale de l'histoire de l'immigration
}

Fabrice Grognet

\section{- To cite this version:}

Fabrice Grognet. Comment concilier l'inconciliable: la place concevable de l'ethnologie dans le musée de la Cité nationale de l'histoire de l'immigration. Museum International, 2007, 59 (1-2), pp.49-56. 10.1111/j.1755-5825.2007.0107x.x . hal-01882404

\section{HAL Id: hal-01882404 https://hal.science/hal-01882404}

Submitted on 3 Oct 2018

HAL is a multi-disciplinary open access archive for the deposit and dissemination of scientific research documents, whether they are published or not. The documents may come from teaching and research institutions in France or abroad, or from public or private research centers.
L'archive ouverte pluridisciplinaire HAL, est destinée au dépôt et à la diffusion de documents scientifiques de niveau recherche, publiés ou non, émanant des établissements d'enseignement et de recherche français ou étrangers, des laboratoires publics ou privés. 


\title{
Comment concilier l'inconciliable : la place concevable de l'ethnologie dans le musée de la Cité nationale de l'histoire de l'immigration
}

\author{
Fabrice Grognet
}

Résumé : A l'origine de leur relation en France, musée et ethnologie ont besoin l'un de l'autre. La recherche s'intéresse alors aux sociétés «sans histoire » et le musée archive l'altérité supposée disparaître entre les peuples. Mais après la seconde guerre mondiale, la recherche ethnologique se développe et se redéfinit comme une science des cultures «vivantes » dans d'autres institutions. Dès lors le divorce semble consommé entre la science tournée vers le contemporain et le musée « sauvegarde » du passé. Pourtant, l'ethnologie, comme entreprise de collecte, ne peut-elle pas répondre aux impératifs mémoriels ? La nouvelle Cité nationale de l'histoire de l'immigration pourrait devenir à terme ce lieu de dialogue entre histoire et ethnologie.

Née dans les musées du XIX ${ }^{\mathrm{e}}$ siècle autour de l'élaboration de collections permettant d'archiver l'altérité supposée disparaître, l'ethnologie s'est peu à peu affranchie du modèle des sciences de la nature pour se redéfinir à partir de nouvelles sources et suivant un axe qui lui est propre : l'observation du contemporain.

A la collecte des «objets témoins », envisagés comme les archives objectives de l'humanité, s'est donc substituée celle du témoignage oral, de la photographie et du film, et la quête illusoire des traditions originelles s'est métamorphosée en étude des sociétés d'aujourd'hui.

Entre le musée conservatoire figeant le passé en une «mémoire d'Etat » (Prado, 2003) et la démarche ethnographique, processus continu d'observation des identités en mouvement, le divorce semble consommé. Comme l'a écrit Jean Jamin, « l'étonnant est que l'anthropologie ne s'en est pas portée plus mal. Et c'est d'un œil parfois amusé, parfois attendri, souvent 
indifférent, que les chercheurs et enseignants regardent ce qui n'est plus désormais, que le passé de leur discipline » (Jamin, $1998: 66)$.

Dès lors, quel peut être l'apport de l'ethnologie dans une Cité nationale de l'histoire de l'immigration et en particulier dans son musée? N'y a-t-il pas un paradoxe, presque un malentendu, à vouloir associer l'ethnologie à une institution de mémoire ? L'ethnologie, comme entreprise de collecte, peut-elle répondre aux impératifs mémoriels ? Le musée peut-il, quant à lui, répondre aux questions d'aujourd'hui et à la patrimonialisation du contemporain ?

\section{De l'objet « témoin » au témoignage de l'Homme ; du musée au terrain ; du passé au présent}

Historiquement, l'ethnologie s'est institutionnalisée en France à la fin du XIX ${ }^{\mathrm{e}}$ siècle dans ces «lieux de mémoire » (Nora, 1997) par excellence que sont les musées. Lorsque le premier musée qualifié d'ethnographique est créé en France en 1878 suite à l'exposition universelle de la même année, la définition même de l'ethnographie semble s'orienter vers une description des productions matérielles des peuples «primitifs » et «sans histoire ${ }^{1}$. Pourtant les collections constituant le fond du tout jeune Musée d'Ethnographie du Trocadéro montrent que le qualificatif «d'ethnographique » recoupe, certes une majorité d'objets appartenant à des peuples «exotiques » contemporains, mais aussi des vestiges archéologiques de civilisations disparues, des fac-similés (à échelle réelle ou en modèles réduits), des restes humains, ou encore des représentations de types physiques réalisées à partir de sculptures, de moulages ou de photographies. En fait, l'entreprise de description des peuples est alors subordonnée à l'histoire naturelle de l'Homme, et toutes les choses matérielles chargées de retranscrire la

\footnotetext{
${ }^{1}$ Le moraliste suisse Alexandre César de Chavannes (1731-1800) dans son Essai sur l'éducation intellectuelle avec le projet d'une science nouvelle emploie en 1787, et pour la première fois en français, le terme « d'ethnologie » pour le discours sur les mœurs et coutumes des peuples non occidentaux.
} 
longue marche de l'humanité vers la «civilisation » 2 sont qualifiées de manière générique d'objets ethnographiques.

Toujours est-il que l'étude des peuples et l'ambition de conserver toutes traces matérielles présentant une altérité, tant physique que culturelle, tant présente que passée, semblent alors intimement liées. Le musée devient dès lors le légitime laboratoire des «anthropologues de cabinet $\gg^{3}$.

En même temps que s'élaborent les théories à partir des crânes et des objets mis en série, les collections ethnographiques participent également à l'élaboration d'un patrimoine national français à partir d'éléments culturels provenant principalement de l'étranger ou des colonies, mais également des campagnes françaises métropolitaines. En effet, si l'acception la plus courante dans un contexte d'expansion coloniale insinue alors que l'ethnologie s'intéresse de manière privilégiée aux peuples dits «sauvages », l'étude du «folklore » et des traditions rurales européennes implique qu'aucune partie du monde n'est à l'abri de devenir l'objet de la jeune science. L'étude de «l'aborigène » australien (considéré comme le plus «primitif des sauvages ») ou du breton de métropole relève finalement du même effort fondamental, à savoir l'archivage de ce qui est supposé en train de disparaître : le «sauvage» du fait de la colonisation ; le « rural » du fait de l'industrialisation.

Ainsi l'ethnologie, discipline de l'urgence à l'objet présumé volatil, s'est avant tout institutionnalisée dans le lieu privilégié du musée comme une science tentant de déjouer l'entreprise destructrice du temps en archivant l'Homme et ses productions.

\footnotetext{
2 Suivant la conception linéaire de l'évolution des sociétés humaines de l'époque (Auguste Conte, Lewis Henry Morgan), les productions des sociétés «primitives » sont capables d'expliquer, par comparaisons, le passé des sociétés occidentales envisagées comme l'aboutissement de l'histoire du progrès humain.

${ }^{3}$ Les « anthropologues de cabinet » théorisent au musée, à partir de récits de voyages et d'objets ramenés, le plus souvent des colonies, par les naturalistes, médecins, militaires ou administrateurs coloniaux.
} 
Il faut attendre la professionnalisation finalement tardive de l'ethnographie en France au cours des années $1930^{4}$ pour qu'une définition autonome (vis-à-vis de l'histoire naturelle) de l'objet ethnographique soit établie à partir de l'influence théorique du socio-anthropologue Marcel Mauss (1872-1950). L'ethnographie cesse alors d'être la description exhaustive des peuples à travers l'histoire de l'humanité. Elle recouvre désormais l'étude culturelle par le biais de l'observation directe, à partir d'une situation empiriquement constituée. Dès lors l'ethnologie devient l'étude de cultures «vivantes » avec comme principe méthodologique l'observation participante. Le scientifique ne peut plus se contenter d'étudier et de classer les productions matérielles dans le cadre du musée qui demeure le seul lieu institutionnel de recherche. L'ethnologue, par définition «de terrain », remplace alors l'anthropologue de cabinet. Cette nouvelle façon de procéder est mise en pratique sans que les productions matérielles ne perdent pour autant leur valeur heuristique prédominante. Les objets les plus couramment employés au sein des sociétés demeurent des « témoins » scientifiques, suivant la formule consacrée par les Instructions sommaires pour les collecteurs d'objets ethnographiques: «Presque tous les phénomènes de la vie collective sont susceptibles de se traduire par des objets donnés, à cause de ce besoin qui a toujours poussé les hommes à imprimer à la matière la trace de leur activité. Une collection d'objets systématiquement recueillis est donc un riche recueil de «pièces à conviction », dont la réunion forme des archives plus révélatrices et plus sûres que les archives écrites, parce qu'il s'agit ici d'objets authentiques et autonomes, qui n'ont pu être fabriqués pour les besoins de la cause et caractérisent mieux que quoi que ce soit les types de civilisation »(Instructions, $1931: 6-7)$.

L'ethnographie devient officiellement une affaire de spécialistes de «l'étude de la civilisation matérielle » (ibid: 5).

\footnotetext{
${ }^{4}$ Dans le Musée d'Ethnographie du Trocadéro (MET) dans un premier temps, puis au Musée de l'Homme et au Musée National des Arts et Traditions Populaires à partir de 1937, ces deux derniers musées naissant de la séparation du fond du MET concernant l'ethnographie de la France et celle du reste du monde.
} 
Le matériel, le concret, l'objectif, est la clef qui permet d'atteindre d'autant mieux les fondements immatériels d'une culture que l'ethnologue se méfie du témoignage d'un « indigène » dont il ne connaît pas la langue et qu'il rémunère le plus souvent. L'objet tangible, document préexistant à la collecte, demeure donc la source privilégiée. Le musée reste lui aussi, encore pour un temps, l'institution de prédilection et le seul pôle institutionnel où il ne s'agit plus exclusivement de «rassembler des objets, mais aussi et avant tout de comprendre les hommes, et moins d'archiver des restes desséchés comme dans un herbier, mais de décrire et analyser des modes d'existences auxquels l'observateur prend directement part », selon les mots de Claude Lévi-Strauss (Lévi-Strauss, 1958 : 404).

Avec le développement de la méthodologie de terrain (apprentissage notamment de la langue vernaculaire), l'apport de la photographie et du cinéma, les productions matérielles perdent de plus en plus de leur valeur heuristique. Plus que les objets, c'est l'Homme-objet d'étude qui reprend ses droits, et l'ethnologue s'intéressant à l'élaboration, à la persistance, à la redéfinition des identités des groupes sociaux dont il est le témoin, produit de nouvelles sources.

L'émergence de nouvelles institutions sans collections (universités, Ecoles des Hautes Etudes) ainsi que la décolonisation font que les ethnologues délaissent de plus en plus le musée qui a vu naître et se développer leur discipline. Sous l'influence également du paradigme structuraliste, la recherche ethnologique se désintéresse des objets et part s'installer et se développer dans des institutions où le substitut d'objet peut suffire ${ }^{5}$. Parallèlement à cet éloignement ou abandon de l'ethnologie pour ses collections (Ségalen, 1996), pour ce qui devient finalement son histoire (Jamin, 1998), le «musée laboratoire » ethnologique, qu'il soit destiné à présenter les cultures populaires françaises (Musée National des Arts et Traditions Populaires) ou les cultures étrangères (Musée de l'Homme), s'essouffle. Les galeries publiques ne sont pas rénovées et le

\footnotetext{
5 «Les ethnologues de ma génération ont encore collecté quelques objets : j’ai rapporté au musée une caisse d'outils de pierre de chez les Baruya de Nouvelle-Guinée. Mais cela a été ma dernière occasion. Ensuite, je n'ai fait que des films, comme la plupart de mes collègues » (Godelier, 1999 : 19).
} 
musée se fige. Il ne présente plus la science en train de se faire, mais témoigne plutôt des modes de vie dits «traditionnels », «préindustriels », pétrifiés « dans un éternel présent » (Gaugue, $1999: 337)$.

L'institution, en ne renouvelant pas ses collections exposées, en fétichisant par exemple l'objet de l'époque coloniale collecté dans la crainte d'une probable disparition de l'altérité6, fait ainsi renouer artificiellement en galerie publique l'ethnologie avec son ancienne acception du XIX ${ }^{\mathrm{e}}$ siècle.

\section{Le possible dialogue entre mémoire et ethnologie}

L'ethnologie en a-t-elle pourtant fini avec le monde des musées et le processus de patrimonialisation?

Comme on vient de le voir, l'ethnologie d'aujourd'hui ne semble plus une science capable de soutenir à elle seule le projet d'un musée dont l'idée (conserver et présenter des objets qui ne sont plus utilisés ${ }^{7}$ n'a guère évoluée depuis le $\mathrm{XIX}^{\mathrm{e}}$ siècle. Comment d'ailleurs collecter le contemporain 8 qui suggère un processus continu, et surtout, pourquoi le faire, alors que suivant une idée couramment répandue, on assisterait à une uniformisation des cultures?

Pourtant depuis les années 1970, la France ne cesse de se doter d'institutions (écomusées, musées régionaux et industriels) au «patrimoine ethnologique» proclamé. Société de « consommation », la France, consciente de ses propres processus continus de changements historiques, devient une « société de conservation » (Fabre, 1994).

\footnotetext{
${ }^{6}$ Comme le montre le premier musée national français du nouveau millénaire : le Musée du Quai Branly

7 En France, les musées se sont surtout développés avec la tutelle de l'Etat et autour des collections de beaux-arts.

8 Voir Le Menestrel (1996).
} 
La prise en considération d'un patrimoine lié à l'immigration en France est même clairement formulée, dès le début des années 1990, pour une ouverture possible du champ des musées d'ethnographie' .

Mais cette collecte et mise en patrimoine d'objets de «toutes sortes, comme autant de témoignages d'un passé révolu ou d'une culture laminée, obéit à une logique qui lui est propre et qui, à mon sens, n'entretient pas de relation nécessaire avec l'ethnologie comme science sociale (...) Ce ne sont pas des faits sociaux qui sont ainsi rassemblés mais des objets précieux que collecteurs et spectateurs reconnaissent immédiatement, avec émotion et plaisir » (Fabre, 1986).

Autrement dit, collecter des objets pour un musée, même consacré à des groupes sociaux déterminés, n'est pas nécessairement faire œuvre d'ethnographie, de même que regrouper dans un musée les anciennes sources de l'ethnologie ne créé pas de fait un musée d'ethnographie au sens moderne du terme ${ }^{10}$.

Pour que l'objet collecté devienne une source ethnographique, un objet « témoin » sur le plan scientifique, il convient que l'ethnologue sélectionne, parmi les productions matérielles d'une société, celles qui seront interprétées dans « cette aventure singulière qu'est la relation du chercheur et du groupe qu'il a choisi » (Fabre, ibidem) comme ressource pour sa recherche. L'objet ethnographique n'existe donc pas sans le regard et l'analyse de l'ethnologue.

Mais un objet ethnographique le reste-t-il ? Si l'on part du principe que «l'ethnologie a pour fondement l'étude des milieux contemporains, des cultures vivantes » (Pizzorni-Itie, 1996), un objet cesserait d'être ethnographique, c'est-à-dire d'intéresser les ethnologues, à partir du moment où il ne serait plus utilisé. L'objet sans utilité pratique ou symbolique ne serait plus un référent pour les membres du groupe, et donc ne pourrait être leur témoin. Autrement dit, l'objet

\footnotetext{
9 Voir Guibal (1992: 159) et Pizzorni (1996 : 244).

10 Le Musée du Quai Branly acquiert ses nouvelles collections en salles des ventes sans pratiquer de collecte sur le terrain.
} 
ethnographique serait périssable. Il mourrait en même temps que cesse toute pratique culturelle le concernant. D'une certaine manière, l'objet serait alors évacué de la culture qui l'a créé. Il pourrait demeurer un témoignage, un document culturel comme en archéologie, mais il ne serait plus que la preuve de pratiques éteintes.

Pourtant, derrière l'objet ethnographique qui mute avec le temps en document historique, on devine une possible rencontre entre histoire et ethnologie autour de l'élaboration du patrimoine. En ayant pour objet les processus identitaires, l'ethnologie n'est pas coupée de l'histoire et de l'archivage des faits humains. Si l'historien part du fait retrouvé dans les archives pour remonter le cours du temps jusqu'au présent, l'ethnologue quant à lui part de son observation pour retourner dans le passé11. En suivant l'un et l'autre leur parcours respectif, nul doute que l'historien et l'ethnologue peuvent se rencontrer dans un lieu finalement inattendu au regard de l'évolution de la discipline : le musée.

Mais de quel musée parle-t-on alors ? Du musée traditionnel qui n'envisage que le « sauvetage de mémoire », ou du musée «d'interprétation », de «société » (Duclos, 1992), qui met en question le contemporain?

Aussi, de la définition du musée dépend la manière spécifique d'envisager sa collection.

\section{Le musée de la Cité nationale de l'histoire de l'immigration}

Les galeries permanentes du musée de la Cité nationale envisagent de produire la synthèse historique de deux cents ans d'immigration en France au moment même où ce processus est un des thèmes majeurs du débat politique actuel. Aussi, cette histoire peu valorisée jusque-là, ce fait social d'un intérêt finalement récent tant chez les historiens que chez les ethnologues en France, devient le sujet d'une nouvelle institution qui a pour but de s'adresser aux citoyens

\footnotetext{
11 «l'ethnologue, voué à l'étude de sociétés vivantes et actuelles ne doit pas oublier que pour être telles, il faut qu'elles aient vécu duré et donc changé » (Lévi-Strauss, 1958 : 132).
} 
d'aujourd'hui en réaffirmant la place de l'immigration dans l'histoire de la République (Raffarin, 2003).

«Centre d'histoire et de mémoire vivante, à vocation culturelle » (Toubon, 2004 : 10), la Cité nationale, dont le musée ne possède pas de collections préexistantes, envisage également d'établir l'inventaire du patrimoine de l'immigration à travers un « appel à collecte » national qui mobilise directement la société civile (associations et particuliers). Autrement dit, pour constituer son fond propre qui trouvera une visibilité tant dans la galerie permanente (autour de thématiques préalablement déterminées) que dans la « galerie des dons » 12 , le musée sollicite directement les migrants et leurs descendants. Les objets spontanément donnés représentent finalement ce que les acteurs (ou leurs descendants) envisagent comme étant représentatif de leur propre parcours de migrants.

Mais tous ces objets et témoignages ainsi réunis constituent-ils une collection cohérente ou resteront-ils une somme de mémoires individuellement exprimées ? Rentreront-ils tous dans le patrimoine national français ou opèrera-t-on une sélection ${ }^{13}$ et suivant quels critères ? Quelles catégories socio-culturelles de migrants répondront finalement à cet appel ? Et fondamentalement, rassembler des dons est-il véritablement faire acte de collecte ?

Plus que le parcours permanent (ancré dans un discours historique) et la «galerie des dons » (participation et appropriation de l'espace public par les migrants), l'élaboration de futures expositions temporaires ${ }^{14}$ touchant à des thématiques d'actualité semblent pouvoir être l'occasion de rassembler des documents scientifiques concrets et nouveaux au cours de recherches sur le terrain. Une collecte ethnographique (récits de vie, photographies, films,

\footnotetext{
12 Dans sa mission de sauvegarder les traces matérielles et immatérielles de l'histoire de l'immigration, la Cité doit s'appuyer sur la participation des immigrés en France. C'est dans ce but qu'a été créée une "galerie des dons $»$.

13 «Collectionner est par définition une démarche sélective qui exclut toute neutralité. Elle implique toujours une certaine discrimination, qui résulte d'une interprétation du passé comme du présent » (Le Menestrel, 1996 : 80).

${ }^{14} \mathrm{Au}$ regard de l'histoire des musées traitant des cultures et donc des identités, il apparait que seule une politique dynamique d'expositions temporaires associée à la présentation d'une « exposition de synthèse » (Guibal, 1992) semble pouvoir contrebalancer la cristallisation des cultures qu'engendrent les galeries dites permanentes.
} 
objets) pourrait ainsi permettre d'établir un inventaire des choses (matérielles et immatérielles) mobilisées, consciemment ou non, par les migrants d'aujourd'hui.

Le nouveau corpus ainsi rassemblé permettrait de traiter la dimension contemporaine, à côté du traitement artistique ${ }^{15}$, sans se limiter à la seule dimension spontanée du récit, et de compléter, voire d'éclairer sous un jour nouveau, le rassemblement d'objets de la «galerie des dons $»^{16}$. Ce procédé d'acquisition scientifique permettant de renouveler le fond du musée, de porter un regard plus «éloigné » ou distancié sur le phénomène d'immigration, ne fournirait-il pas également à terme des archives mobilisables pour les historiens de demain ?

Mise en ordre du passé historique, participation de la société civile à l'élaboration d'un patrimoine national, questionnement par le biais d'artistes : où se situe la place de l'ethnologie dans la Cité nationale de l'histoire de l'immigration?

Pour l'instant, la question n'est pas encore tranchée dans la jeune institution qui se définie tout en s'élaborant. Toutefois on devine à terme la profitable association, tant pour la discipline ethnologique qui pourrait ainsi (re)valoriser ses recherches actuelles dans un musée national, que pour l'institution du musée aux collections et au patrimoine en devenir.

La nouvelle Cité nationale de l'histoire de l'immigration deviendra-t-elle ce lieu à inventer instaurant un dialogue entre histoire et ethnologie, entre l'étude du passé et l'observation du présent, autour de la constitution d'un nouveau patrimoine?

Le musée de la Cité, par le biais notamment de sa programmation d'expositions temporaires et d'une politique d'acquisition qui lui serait liée, pourrait devenir l'organe de cet échange.

\footnotetext{
15 Le musée possède déjà des collections d'art contemporain.

16 Il serait à cet égard intéressant d'analyser l'éventuel différentiel pouvant exister entre les résultats de « l'appel à collecte » de la CNHI et ceux que procureraient un étude ethnographique autour du même panel de personnes.
} 


\section{Bibliographie}

Jean Copans, 2005, L'enquête ethnologique de terrain, Armand Colin, série l'enquête et ses méthode $», 127 \mathrm{p}$.

Jean-Claude Duclos, 1992, « Pour des musées de l'homme et de la société », in Le débat $\mathrm{n}^{\circ} 70$ mai-août, pp. 174-178.

Daniel Fabre, 1986, «L'ethnologue et ses sources » in Terrain, Numéro 7, «Approches des communautés étrangères en France », pp 3-12.

Daniel Fabre, 1994, «Ethnologie et patrimoine en Europe, conclusions et perspectives du Colloque du Tours », in Terrain $\mathrm{n}^{\circ} 22$, pp.145-150.

Anne Gaugue, 1999, «La mise en scène de la nation dans le musée d'Afrique tropical », in Ethnologie française tome XXIX, pp. 337-344.

Maurice Godelier, 1999, « Un musée pour les cultures » in Sciences Humaines Hors Série n²3, pp. 19-20.

Jean Guibal, 1992, «Quel avenir pour le musée des ATP ?, entretien avec Jean Guibal », in Le débat $\mathrm{n}^{\circ} 70$ mai-août, pp. 157-163.

INSTRUCTION SOMMAIRES POUR LES COLLECTEURS D'OBJETS ETHNOGRAPHIQUES, 1931, Musée d'Ethnographie du Trocadéro, Paris.

Jean Jamin, 1998, «Faut-il brûler les musées d'ethnographie ? », in Gradhiva, 24, pp. 65-69. Sarah Le Menestrel, 1996, « La collecte de l'objet contemporain. Un défi posé au Musée de la Civilisation à Québec », in Ethnologie française, " culture matérielle et modernité », pp.74-91. Claude Lévi-Strauss, 1958, Anthropologie structurale I, Ed Plon.

Pierre Nora, 1997, Les lieux de mémoire, collectif sous la direction de Pierre Nora, trois tomes, Gallimard.

Patrick Prado, 2003, Territoire de l'objet: faut-il fermer les musées ?, ed des archives contemporaines, $149 \mathrm{p}$.

Florence Pizzorni-Itie, 1993, «Réflexions autour d'un paradoxe : faut-il et comment traiter du contemporain dans les musées d'ethnographie "), in Actes des premières rencontres européennes des musées d'ethnographie, Ecole du Louvre, pp. 243-249.

Jean-Pierre Rafarin, 2004, «Lettre de mission du Premier ministre », in Mission de préfiguration du Centre de ressources et de mémoire de l'immigration, La documentation française, $254 \mathrm{p}$.

Jacques Toubon, 2004, Mission de préfiguration du Centre de ressources et de mémoire de l'immigration, La documentation française, 254p. 